\title{
Seguridad y Salud en las Prácticas Formativas de Estudiantes de 6 Instituciones Educativas Hospital San José de Popayán. 2016
}

\author{
Health and safety in the training of students of 6 educational institution \\ San José from Popayan hospital practices 2016
}

\author{
Sandra F Muñoz ${ }^{1}$ B, Gloria Gonzales. ${ }^{2}$ \\ ${ }^{1}$ Enfermera magister en Materno Infantil. Profesor asociado. Programa de Enfermería. Universidad del Cauca. \\ ${ }^{2}$ Enfermera magister en Salud Ocupacional. Profesor ocasional. Programa de Enfermería. Universidad del Cauca
}

\section{Resumen}

\section{Introducción.}

El decreto 1072 de 2015 del Ministerio de Trabajo, unificó las políticas y estrategias de formalización y protección a los trabajadores y el 055 de 2015 determinó el cumplimiento de las condiciones de seguridad laboral y su afiliación al sistema general de riesgos laborales para los estudiantes de todos los niveles académicos de instituciones educativas públicas o privadas. El cumplimiento de las condiciones de seguridad y salud de los escenarios de práctica, se evalúa a través de actividades de prevención, higiene y seguridad laboral, mediante la supervisión de elementos de protección personal y el análisis de los puestos de trabajo.

\section{Objetivo.}

Evaluar el cumplimiento de las condiciones de Seguridad en el trabajo de los estudiantes que realizan prácticas formativas.

\section{Método.}

Descriptivo transversal. Muestreo probabilístico a 128 estudiantes seleccionados por afijación proporcional de 6 instituciones educativas.

\section{Resultados.}

El 64\% (82) de los estudiantes pertenecen al género femenino, en formación profesional de pregrado en un $63 \%(81)$, postgrado en un $27 \%(35)$ y tecnológico en un $9 \%(12)$. Los médicos internos y de postgrados, laboran 264 horas al mes, sin embargo el 31\% (40) expresan no tener descanso. El 50\% de los estudiantes no realizan procedimientos, el $87 \%$, manifiestan conocer los riesgos a los que están expuestos y solo un $4 \%$ de los estudiantes reporto haber sufrido un accidente laboral asociado a riesgos biológicos. Se encuentra significancia estadística $(p=0,004)$ entre el tipo de actividad y el riesgo laboral.

\section{Conclusiones:}

Evaluar las condiciones de seguridad laboral, fortalece los procesos de autocuidado.
Palabras claves. DeCS, Salud laboral, Prácticas clínicas.

\section{Abstract}

Introduction. 2015 of the Ministry of Labour Decree 1072, unified policies and strategies of formalization and protection of workers and the 0552015 determined compliance with occupational safety conditions and its affiliation to the general system of risks employment for students of all academic levels of public or private educational institutions. The fulfilment of the conditions of safety and health practice scenarios, is evaluated through prevention, hygiene and occupational safety by monitoring items of personal protection and the comprehensive analysis of the positions of work.

\section{Objective.}

Evaluate the fulfilment of the safety conditions in the work of students who perform formative practices.

\section{Methods.}

Transversal descriptive. Probabilistic sampling to 128 students selected by proportional affixation of 6 educational institutions.

\section{Results.}

The $64 \%(82)$ of the students belong to the feminine gender in training of undergraduate in a $63 \%(81)$, postgraduate diploma in $27 \%$ (35) and technological in 9\% (12). Physicians postgraduate courses and internal working 264 hours per month, however $31 \%$ (40) express not have rest. $50 \%$ of the students do not perform procedures, $87 \%$, they appear to know the risks to which they are exposed and only $4 \%$ of students reported having suffered an accident at work associated with biological hazards. Is statistical significance $(p=0,004)$ between the type of activity and labor risk. Conclusion: Evaluate de conditions of occupational safety, strengthens the processes of self-care.

Key words. DeCS, Occupational health, clinical practice. 


\section{Introducción}

El Sistema General de Riesgos Profesionales, es el conjunto de entidades públicas y privadas, normas y procedimientos, destinados a prevenir, proteger y atender a los trabajadores, de los efectos de las enfermedades y los accidentes que puedan ocurrirles con ocasión ó como consecuencia del trabajo que desarrollan.[1] Con la aplicación del decreto 1443 de 2014, se implementa en Colombia, el Sistema de Gestión de la Seguridad y Salud en el Trabajo (SGSST), como una herramienta de gestión, para mejorar la calidad de vida laboral de los trabajadores. Este decreto basa su aplicación en la generación de una cultura de seguridad articulada con la productividad, desarrollo del talento humano, gestión de calidad, mejoramiento de procesos, condiciones adecuadas de puestos de trabajo, prevención e intervención de riesgos y peligros asociados a las condiciones laborales o profesionales; procurando el bienestar físico y mental, de todas aquellas personas que se encuentran vinculadas en calidad de trabajadores, contratistas, sub contratistas como también de colaboradores (estudiantes ) con quienes se tiene una relación docencia servicio.

La regulación del Sistema General de Riesgos Profesionales, se inicia en Colombia, con el decreto 1295 de 1994, posteriormente el decreto 2376 de 2010, reglamenta la relación docencia -servicio para los programas de formación del Talento Humano del área de la salud y específica sobre las garantías de seguridad, protección y bienestar de los estudiantes[2]. El decreto 055 de 2015 del Ministerio de Salud y Protección social, cataloga como afiliados obligatorios al sistema general de riesgos Laborales, a los estudiantes de todos los niveles académicos de instituciones educativas públicas o privadas, fija el tipo de exposición y establece que los escenarios de práctica, supervisen y verifiquen el cumplimiento de las condiciones de seguridad laboral. Para evaluar estas condiciones, se deben caracterizar los riesgos laborales y determinar las condiciones de prevención, higiene y seguridad laboral según programa y tipo de actividad como también determinar el conocimiento de riesgos en los estudiantes. El seguimiento de estas condiciones, facilita el reconocimiento de prácticas de autocuidado, adherencia a las guías y protocolos en la comunidad académica.

\section{Metodos}

Estudio de tipo descriptivo de corte transversal, permitiendo evaluar el cumplimiento de las condiciones de Seguridad en el trabajo de los estudiantes de seis instituciones activas con quienes el Hospital tiene una relación docencia servicio. La población objeto del estudio está conformada por 700 estudiantes. La muestra se definió mediante un muestreo probabilístico aleatorio simple, aplicando la fórmula para poblaciones finitas con un margen de error del $5 \%$ un nivel de confianza del $96 \%$, para un total de 128 estudiantes distribuidos por afijación proporcional por cupo asignado, a cada programa. Teniendo como criterios su deseo de participar y realizar su práctica formativa, en el Hospital y como criterio de exclusión, el abandono al mismo.
Para identificar las condiciones de seguridad y salud y priorización de riesgos laborales, se utilizaron los cuestionarios: "Análisis integral de puestos de trabajo metodología afro" que permite indagar a profundidad en los procesos, procedimientos, tareas y actividades para identificar las causas básicas que afectan el desempeño del personal y el de "Inspección de Elementos de Protección Personal en Estudiantes" de la ARL Positiva, Gestor Documental, previo consentimiento de propiedad intelectual de la Administradora de Riesgos Laborales Positiva. El sistema de información y seguimiento de los riesgos, se realizó mediante una matriz de peligros por puesto de trabajo según la norma GTC45 ICONTEC, versión 2012. La recolección de la información se realizó en las siguientes etapas.

1. Presentación del anteproyecto ante los programas de las instituciones educativas, el Comité de Ética de investigación y la Coordinación de Salud y Seguridad en el Trabajo del Hospital Universitario San José.

2. Información a los estudiantes encuestados sobre el objetivo del proyecto de investigación, los beneficios y/o riesgos, sobre su participación, condiciones y socialización de resultados.

3. Determinación del sitio de aplicación, se realizó según profesión u oficio en el área, tomando una sola muestra por área, hasta terminar la muestra.

Para el análisis de datos, se tabularon los datos del cuestionario análisis integral de puestos de trabajo - metodología afro y se consignaron en el paquete estadístico SPSS versión 2013. Se realizó un análisis univariado, en el caso de las variables cualitativas, se calcularon distribución de frecuencias y porcentajes; para las variables cuantitativas se incluyeron medidas de tendencia central como promedios. Se determinó la asociación de variables, mediante el chic cuadrado.. Una vez finalizado el análisis, la información se consolidó en tablas y gráficos.

Para el desarrollo de este proyecto de investigación se tuvo en cuenta las pautas éticas CIOMS, Ginebra 2002. Las investigadoras consideran que las condiciones de Seguridad y Salud en el trabajo de los estudiantes que realizan prácticas formativas, generara beneficios para los programas de promoción y prevención de la salud ocupacional orientados hacia el manejo de riesgos psicolaborales, previa revisión y aprobación por parte del Comité de Ética del Hospital Universitario San José. Siguiendo lo establecido en el informe Belmont (1978) La investigación se basó en los principios y valores éticos de respeto por las personas, el principio de beneficencia, la justicia, como principio que garantizara que no existirá ningún tipo de sesgo o discriminación de género, etnia, programa, a quienes participen en este proyecto, como también se informará que los beneficios que se logren serán dirigidos a todos los participantes, según los hallazgos.

La participación voluntaria de los sujetos de estudio se hizó evidente en la firma del documento del consentimiento informado, con el fin de garantizar el cumplimiento de su voluntad y el respeto de ésta. Para promover la confidencialidad de la información de los cuestionarios, se utilizó un sistema 
de código aplicado. Tanto los formatos diligenciados como la hoja con los códigos permanecieron bajo la responsabilidad de una de las investigadoras. Se dejó explícito que ningún funcionario tendrá acceso a los formatos diligenciados, este parámetro obedece a los planteamientos mencionados en la Declaración de Helsinki de la Asociación Médica Mundial que tiene como pilar fundamental de una investigación ética resguardar la intimidad de la persona y la confidencialidad de su información personal.

\section{Resultados}

Se evaluaron 128 estudiantes $64 \%$ (82) del género femenino y $36 \%(46)$ del género masculino, en formación profesional de pregrado de medicina, enfermería, fonoaudiología, fisioterapia y bacteriología en un $63 \%(81)$, postgrado de medicina, en un $27 \%(35)$ y tecnológico en un 9\%(12). Distribuidos en áreas de internación en un 54\%(69), ambulatorias en un 15\%(19), urgencias adultos y pediátricos en un $12 \%$ (15), cirugía, endoscopia en un 9\%(12) y el 3\%(4) en laboratorio clínico y patología. Con una antigüedad en el sitio de práctica, de 5 días para pregrado, 30 días para internado y 90 días para postgrado.

Al realizar seguimiento en su seguridad laboral, se observa que, el $53 \%$ tienen turnos diurnos, el $16 \%$ mixtos, el $14 \%$ nocturnos, el $10 \%$ con un turno adicional y el $9 \%$ no respondió. Gráfico 1. Según el tipo de actividad y frecuencia, la consideran mixta en un $56 \%$; manual en un $41 \%$ y mecanizado el $3 \%$. Gráfico 2 En cuanto al tipo de turnicidad, el $76 \%$ de las prácticas son diurnas, y el $24 \%$ nocturnas con un máximo 12 horas laboradas para estudiantes de pregrado y postgrado. La oportunidad de descanso durante las rotaciones y/o turnos es de media hora en el $51 \%, 1$ hora en el 36\%,15 minutos en el 13\%. El número de horas laboradas descritas en los cuadros de turno es de 264 horas, sin embargo, los estudiantes de postgrado manifiestan no tener descanso pre turno y pos turno durante las rotaciones en un $31 \%$ (40); el $45 \%$ refieren tener un día libre al mes, el $37 \%$ dos días, el $14 \%$ tres días y el $4 \%$ manifiestan no tenerlos. El 23\% tiene más de 6 turnos o guardias, el 16\% realizan hasta 4 turnos y el $8 \%$ les extienden un turno adicional al mes. Al analizar los puestos de trabajo, solo el $50 \%$ de los estudiantes realizan procedimientos clasificados como moderados en un $39 \%$, livianos en un $33 \%$ y sedentarios en un $28 \%$.

\section{FIGURA 1}

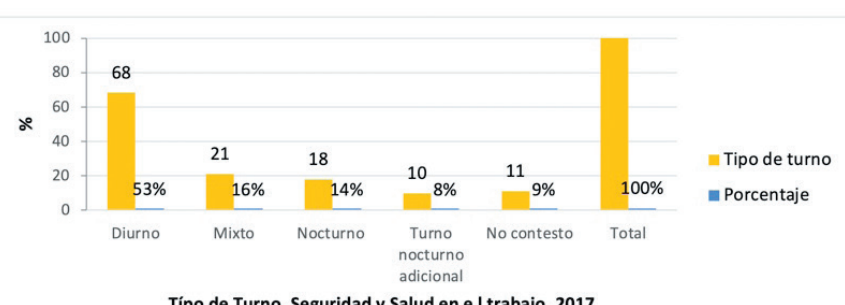

FIGURA 2

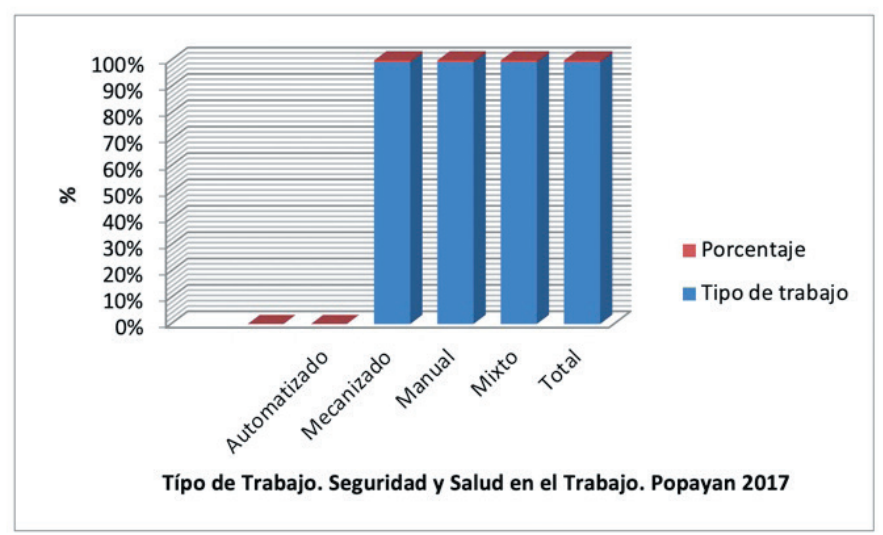

Entre los requisitos de obligatorio cumplimiento en el escenario de práctica se encuentra la inducción virtual, donde se abordan temas de seguridad y salud en el trabajo, rutas de evacuación, seguridad del paciente, humanización y normatividad vigente. Los docentes en sus sitios de práctica, complementan a los estudiantes con temas de seguridad en el paciente, donde el 93\% (119) de los estudiantes, expresan haber recibió inducción inicial en el sitio de trabajo, considerándola efectiva en un 77\% (99). A los médicos internos y residentes, que realizan prácticas permanentes en el Hospital, se les hace entrega de material de protección personal como monogafas, uniformes, gestión de la historia clínica y asignación de sitios de descanso. Al tener en cuenta los estándares de riesgo ocupacional, el Hospital cuenta con una política de seguridad y salud en el trabajo, y entre sus indicadores se encuentran los accidentes laborales, causados por la condiciones en el trabajo en un $4 \%$ y eventos adversos propios de la práctica profesional con un porcentaje del $0 \%$.

\section{Descripción de la Actividad}

Al evaluar la exposición a riesgos durante las prácticas, el 87\% (111) de los estudiantes manifestaron conocer los riesgos, describiendo que en un 38\% son de origen biológico, $36 \%$ biomecánico, $14 \%$ químico, $5 \%$ Psicolaboral y físico en un $7 \%$. La actividad más frecuente para todos los programas es la valoración física en un 63\%; Entre las actividades del programa de Medicina, se encuentran recibo de turno en un $79 \%$; evoluciones en un $96 \%$; respuesta a interconsulta $14 \%$, triage $4 \%$ y atención del parto en un $10 \%$. En el programa de enfermería el $27 \%$ describen realizar actividades de confort, como baño y arreglo de la unidad del paciente, el $25 \%$ toma de signos vitales, el $15 \%$ venopunciones, el $12 \%$ control de líquidos, el $11 \%$ disponen y administran medicamentos; El programa de Fisioterapia realiza terapias respiratorias en un $13 \%$ y actividades de rehabilitación en un $9 \%$. El programa de Fonoaudiología describe actividades de educación y valoración en un $55 \%$. Sólo el $4 \%$ informo haber presentado un accidente de trabajo durante sus prácticas. Al evaluar el puesto de trabajo, los factores de riesgo identificados fueron: biológicos en un 76\%(59) biomecánico y psicosocial en un $10 \%(7)$, químico en un $21 \%(16)$; físico en un $9 \%(7)$; y de seguridad en un $12 \%$ (9); Gráfico 3. Las actividades son consideradas como moderadas y livianas en un 49 y $33 \%$ y sedentarias en un $18 \%$. 
FIGURA 3.

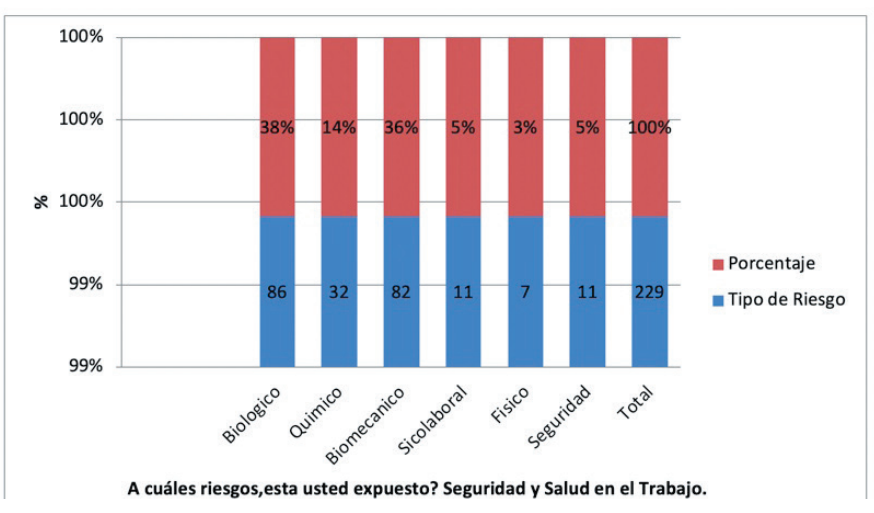

Medios de Trabajo

La política de infraestructura y tecnología segura, del Hospital, se apoya en lineamientos como, la renovación de equipos, tecno vigilancia, mantenimiento, monitorización, tecnología segura y disponible. En el Escenario de práctica, se encuentran equipos, máquinas para realizar actividades y procedimientos al paciente como: bombas de infusión, electromiografía, incubadoras, computadores, lámparas de fisioterapia, monitores de signos vitales, electroterapia, bandas sin fin, caminadora y bicicleta eléctrica, provee de materiales de trabajo como termómetros, fonendoscopios y tensiómetros e instrumental quirúrgico. Sin embargo los estudiantes desconocen el manual de uso de los equipos aunque este se encuentra en las diferentes áreas.

Dependiendo de los requerimientos de seguridad, el Hospital cuenta con elementos de protección en los servicios, como guantes, caretas, delantales de protección, ropa para cirugía, excepto las monogafas, que son de uso personal y son requerimiento para el inicio de práctica. Entre las actividades de promoción y prevención, que se realizan en la inducción a médicos internos y residentes, se les hace entrega y énfasis en el uso y cuidado de las monogafas. El número de procedimientos de enfermería, los sitúa en un mayor riesgo, seguido por profesión de medicina, en las prácticas de cirugía y urgencias y prácticas respiratorias en fisioterapia.

\section{Uso de elementos de protección personal.}

En cada una de las áreas del Hospital, se cuenta con elementos de protección personal como guantes, tapabocas y caretas, para uso de funcionarios y colaboradores que realicen procedimientos. El reglamento de prácticas, exige tener las monogafas en el área de práctica, sin embargo el $71 \%$ de los estudiantes no las portan, el $31 \%$ las portan y las usan y el $26 \%$ las portan y no las usan. La supervisión de las practicas, se realiza, mediante una lista de chequeo, con ítems, que verifican el número de estudiantes, segun cupo determinado, supervisión docente, uso de elementos de bioseguridad, adherencia a guías y protocolos, cumplimiento de cuadros de rotación y a través del reporte de funcionarios del Hospital, ante incidentes o quejas.

\section{Correlación de variables}

En cuanto al tipo de actividad y el riesgo laboral, se encuentra una relación estadísticamente significativa $(\mathrm{p}=0,004)$ con un coeficiente de correlación positiva $(\mathrm{r}=0,097)$. Se observa como los riesgos biomecánico y biológico se perciben más en las actividades livianas y moderadas y en las actividades moderadas, se perciben más los riesgos biomecánico biológico y químico. Gráfico 4.

TABLA1. Relación Tipo de Actividad y Exposición al Riesgo.

Seguridad y Salud en el Trabajo. Popayán 2017

\begin{tabular}{lccc}
\hline & Valor & gl & \multicolumn{2}{c}{$\begin{array}{c}\text { Significación } \\
\text { asintótica } \\
\text { (bilateral) }\end{array}$} \\
\hline $\begin{array}{l}\text { Chi cuadrado de } \\
\text { Pearson }\end{array}$ & $33,285^{\mathrm{a}}$ & 15 & 0,004 \\
\hline $\begin{array}{l}\text { Razón de } \\
\text { verosimilitud }\end{array}$ & 34,128 & 15 & 0,003 \\
\hline $\begin{array}{l}\text { Asociación lineal } \\
\text { por lineal }\end{array}$ & 2,757 & 1 & 0,097 \\
\hline
\end{tabular}

\section{Discusión}

La evaluación del cumplimiento de las competencias en el escenario de práctica, se encuentra, orientado a garantizar la adquisición de conocimientos, destrezas y habilidades, requeridas por los estudiantes en cada disciplina. El 90\% de los estudiantes no conocen que tipo de actividades deben realizar para lograr sus competencias, porque aún no existe un formato guía tanto del ministerio de educación, como de las instituciones educativas, que permita normalizar y obtener indicadores de cumplimiento, sólo se llevan bitácoras de cumplimiento para el caso de programas de postgrado. Con el propósito de evaluar el cumplimiento de las actividades de protección y seguridad en las áreas de trabajo y su exposición del riesgo, el Hospital San José de Popayán, asumió las propuestas planteadas por el decreto 2376 de 2010 relacionadas con las garantías de seguridad, protección y bienestar de los estudiantes de postgrado, como alimentación, uniformes, elementos de protección personal, complementado con actividades de promoción y prevención, a través de inducción virtual, esquemas de vacunación, riesgos profesionales.

El tiempo especificado en el decreto 2376 de 2010 y 055 de 2015, cumple con los parámetros expuestos, sin embargo para los estudiantes de postgrado, las actividades diseñadas en su malla curricular no facilitan los descansos que garanticen su seguridad laboral. Para Valero, la actividad continua, con potencial para interrumpir el ciclo sueño-vigilia, es particularmente evidente en los medios hospitalarios. [3]. Estudios evidencian que un sueño adecuado (entre 6 a 8 horas diarias en promedio) se relaciona con una buena salud y la adopción de conductas saludables[4] mientras 
que hábitos de sueño inadecuados pueden indicar un estilo de vida poco saludable y deficiente estado de salud[5]. Los trastornos en los hábitos de sueño generan una sensación de cansancio permanente, que presiona al consumo de sustancias estimulantes del sistema nervioso central para conservar la vigilia y mantenerse alerta, como la cafeína y el alcohol[6]. Para Feo, los efectos principales y más directos del trabajo por turnos, se relacionan con la calidad del sueño, la fatiga y la sensación general de malestar que puedes ser expresada en un sinnúmero de síntomas inespecíficos como calidad del sueño, trastornos gastrointestinales, cardiovasculares, reproductivos, y de salud mental [7]. En España, los horarios atípicos, el trabajo por turnos o trabajo nocturno afectan al $15,7 \%$ de los trabajadores, este tipo de horarios ha aumentado de forma notable sin tenerse en cuenta que la ordenación del tiempo de trabajo es un aspecto que ejerce una enorme influencia sobre la salud global de los trabajadores [8]. Al realizar supervisión las 264 horas laboradas por mes, se observa una ambigüedad conceptual respecto a las horas laboradas durante las prácticas formativas y las actividades académicas, porque sumadas estas actividades, excederían los horarios reglamentarios. Los trabajadores de la salud, se enfrentan a riesgos de accidentes o enfermedades a causa de su ocupación. En su quehacer solo el $4 \%$ refirió haber presentado un accidente laboral de tipo biológico; Los accidentes por pinchazos representan alrededor de dos (2) millones de exposiciones en el mundo cada año. Una cifra alarmante, que puede incrementarse considerablemente si se toma en cuenta que un número elevado de trabajadores no reportan dichos accidentes generando un subregistro, ubicado entre un 30-80 por ciento, según los Organismos Internacionales de Salud[9]. A pesar de tener claras las competencias, actividades y procedimientos durante las prácticas clínicas, el Hospital no tiene definidos en sus procedimientos un ítem, que enumere los elementos de protección personal, que permitan facilitar en los funcionarios y colaboradores que elementos de protección garanticen su seguridad laboral. Estos se encuentran inmensos algunas veces dentro del procedimiento. Como recomendación la coordinación docencia servicio y salud y seguridad en el Trabajo, deben continuar la evaluación de puestos de trabajo, mediante la aplicación de una matriz de riesgos, que permita determinar los elementos de protección personal para cada una de las actividades estipuladas para el logro de las competencias.

\section{Agradecimientos.}

Las autoras expresan agradecimientos al Departamento de Enfermería de la Universidad del Cauca, al área de Seguridad y Salud en el trabajo del Hospital San José y a los estudiantes que participaron en el estudio.

\section{Declaración de conflictos de intereses:}

Ninguno

\section{Bibliografía.}

1. Ministerio de Gobierno de la República de Colombia. Decreto 1295 de junio 22 de 1994. [internet] 2017, [Citado: 9 de Junio de 2017] Disponible https://www.slideshare.net/ potes98/decreto-ley-1295-de-94-sistema-general-de-riesgosprofesionales-23170940

2. Presidencia de la Republica. Decreto 2376 de 2010, articulo 15. [Internet] 2017, [Citado: 6 de Junio de 2017] Disponible en http://www.uco.edu.co/MARCO $\% 20$ LEGAL\%20ORIGINAL/DECRETO\%202376\%202010.pdf

3. Valero H, Lázaro E. Efectos de la turnicidad laboral sobre la calidad del sueño y la percepción de salud. Rev cubana salud trabajo. 2004; 5(1): 10-19.

4. Chen, MY, Wang, EK, Jeng, YJ. Adequate sleep among adolescents is positively associated with health status and health-related behaviors. BMC Public Health,2006; 6:59.

5. Páez C, M, Castaño JJ. Estilos de vida y salud en estudiantes de una Facultad de Psicología: Lifestyles and health of students in a Psychology Program. Psicología desde el Caribe, (25), 155-178[internet] 2010, [Citado: 6 de Junio de 2017] Disponible http://www.scielo.org.co/scielo.php?script=sci arttext\&pid=S0123-417X2010000100008\&lng=en\&tlng=es.

6. Portuondo O, Fernández G, Cabrera A.P. Trastornos del Sueño en Adolescentes. Revista Cubana Pediatría, 2000. 72(1), $10-14$

7. Feo A J. Influencia del trabajo por turnos en la salud y la vida cotidiana. Universidad Javeriana, Bogotá.2007. [Internet] 2008, [Citado: 4 de Junio de 2017] Disponible en: http://www. javeriana.edu.co/biblos/tesis/enfermeria/tesis38.pdf

8. Calera Alfonso A. Área de Salud Laboral. ISTAS. Tiempos y ritmos de trabajo. Incidencias en la Salud. Alzira. Germania, 2004

9. Galíndez Luis, Rodríguez Yuraima. Riesgos Laborales de los Trabajadores de la Salud. Salud de los Trabajadores [Internet]. 2007 Dic [citado 2018 Oct 08] ; 15( 2 ): 67-69. Disponible en:http://www.scielo.org.ve/scielo.php?script=sci_ arttext\&pid=S1315-01382007000200001\&lng=es.

10. Argudín Y. Educación basada en competencias: Nociones y Antecedentes. México D. F.: Trillas. (2005)

11. Jutel $M$, Jaeger $L$, Suck $R$ et al. Allergen specific immunotherapy with recombinant grass pollen allergens. J Allergy Clin Immunol [Internet]. 2005 sep [citado 10 sep 2014]; 116(3):608-13. Disponible en: http://dx.doi. org/10.1016/j.jaci.2005.06.004

12. Ayala L. Legislación en Salud Ocupacional y Riesgos Profesionales. Segunda edición. Ediciones Salud laboral. 2.001. Bogotá.

13. Aguilar J. Administración de la Prevención de Riesgos 
Profesionales. Ed: Quiramac. Medellín .1992

14. Betancourt O. El trabajo y la salud en Revista de la Sociedad Colombiana de Medicina del Trabajo ISSN 1657.2.000. Sep; Vol. 3 No1:35-41.

15. Fuentes E. García A. Impacto de un Modelo de Integración Docente Asistencial en La Formación Profesional y el Campo Clínico. Cienc. Enferm. [Internet]. 2011 Dic [citado 2017 Ene 17]; 17( 3 ): 51-68. Disponible en: http:// www.scielo.cl/scielo.php?script=sci_arttext\&pid=S071795532011000300006\&lng=es. http://dx.doi.org/10.4067/ S0717-95532011000300006.

16. Gonzales C, R. La enfermera como cuidadora de la salud de los trabajadores en actualizaciones en enfermería ISSN. 2.002 sept; Vol.5, No 3:18-22.

17. Universidad del Valle. Vicerrectoría de bienestar universitario sección salud ocupacional. Cartilla de elementos de protección personal. Disponible en: http:// saludocupacional.univalle.edu.co /cartillaepp.pdf.

18. Universidad del Valle. Vicerrectoría de Bienestar Universitario. Sección de salud ocupacional. Subprograma de higiene y seguridad industrial. Guía de elementos de protección personal. [Internet]. 2016 [Citado 7 de julio del 2016]. Disponible en: http://saludocupacional.univalle.edu.co/salud ocupacional/guia.epp.pdf . Fecha de acceso 07/ 07 /2016.

17. Elementos De Protección Personal (EPP), Disponible en: Https://Www.Arlsura.Com/Index.Php/Component/Content/ Article/75-Centro-De-Documentacion-Anterior/Equipos-DeProteccion-Individual-/1194,Sp-3393

19. Universidad del Valle, Cartilla EPP, [Internet]. 2016 [Citado 7 de julio del 2016]. Disponible en: http://saludocupacional. univalle.edu.co/CartillaEpp.pdf, Definición, Página 4.

20. Manual de normas y procedimientos de bioseguridad. Comité de vigilancia epidemiológica (COVE) división de talento humano salud ocupacional. 2003. [Internet]. 2016 Disponible en: http://www.bvsde.paho.org/bvsacd/cd49/gcbioseguridad.pdf

21. Manual de Bioseguridad. Norma Técnica $N^{\circ} 015$ - Minsa / Dgsp - V.01 Lima - Perú. 2004. [Internet]. 2016 Disponible en: http://www.minsa.gob.pe/dgsp/observatorio/documentos/ infecciones/MANUAL\%20DE\%20Bioseguridad.pdf. Fecha de acceso 07/ 07 /2016.

22. El Centro de Recursos del Departamento de Seguros de Texas. División de Compensación para Trabajadores. Servicios Laborales y Médicos, Alcance y Educación. Protección para los ojos. [Internet]. 2016

Disponible en: http://www.tdi.texas.gov/pubs/ videoresourcessp/spstpeyeprot.pdf.

23. Anteojos, monogafas y lentes de seguridad. [Internet]. 2016 [Citado 20 de octubre de 2.016]. Disponible en: http:// suministrosindustrialesrym.com/hello-world/.
24. Matriz elementos de protección personal. [Internet]. 2016 [Citado 07 de julio de 2016].

Disponible en: http://fisicalabsinvestigacion.uniandes.edu. co/archivos/man salud ocup/Anexos,gissa2013/ Matriz, Carpeta, Gestion Uniandes.pdf.

25. Cancer.net. Medine Plus información para su salud. [Internet]. 2016 Disponible en: https://medlineplus.gov/ spanish/ency/article/003337.htm

26. Ministerio de Protección Social. Manual de Comité Paritario de Salud Ocupacional. Ministerio de la Protección Social. Octubre 2007. P 45

27. Cartilla para la Prevención de Riesgos. Positiva Compañía de Seguros. [Citado el 14 de diciembre de 2016]. [Internet]. [Citado 07 de julio de 2.016] Disponible en: https://www.cdc. gov/spanish/niosh/docs/2013-138

28. El Centro de Recursos del Departamento de Seguros de Texas. División de Compensación para Trabajadores. Servicios Laborales y Médicos, Alcance y Educación. Protección para los ojos. [Internet]. 2016 dic Disponible en: http://www.tdi. texas.gov/pubs/videoresourcessp/spstpeyeprot.pdf.

29. Matriz elementos de protección personal. [Internet]. 2016 dic) [Citado 07 julio de 2016] Disponible en: http:// fisicalabsinvestigacion.uniandes.edu.co/archivos/man_salud_ ocup/ANEXOS_GISSA_2013/Matriz\%20E\%20P\%20P\%20 Carpeta\%20Gestion\%20uniandes.pdf 29. Cartilla para la Prevención de Riesgos. Positiva Compañía de Seguros. [Internet]. 2016 ene[Citado 19 de junio de 2016]. Disponible en :https://www.cdc.gov/spanish/niosh/docs/2013-138 sp/ http://www.ehowenespanol.com/significa-ansi-z411991hechos 152848

30. Román O, Señoret SM. La relación docente-asistencial en el nuevo contexto que establece la reforma de salud. Rev Med Chil 2007; 135(2): 251-256. [Internet]. 2016 oct [Citado 19 de junio de 2016].

Disponible en: http://www.scielo.cl/scieloorg/php/reflinks.php ?Refpid=s0717-9553201100030000600010 . 